\title{
POQAS-S: a Novel Programmer-Oriented Online Question Answering System With Semantic Comprehension
}

\author{
Feng Guo, Guangquan Xu*, Ning Zhang, Kaili Qiu \\ Tianjin Key Laboratory of Advanced Networking (TANK), School of Computer Science and Technology \\ Tianjin University \\ Tianjin, China, 300350 \\ *Corresponding author: Guangquan Xu, email: losin@tju.edu.cn
}

\begin{abstract}
To improve the ability and efficiency of acquiring kno wledge for programmers, programmer-oriented online question a nswering system (POQAS) has been advanced recently. However, current POQAS systems are short of semantic sensing ability. To solve this problem, this paper proposed a novel programmer-ori ented online question answering system with semantic comprehen sion (POQAS-S), which is based on the TuringOS. Since semantic comprehension is introduced, our POQAS-S system can underst and the input information more precisely. Further, POQAS-S is a ble to give a more precise answer to users than traditional POQA $S$ systems. In this paper, we developed a concrete POQAS-S syste $m$ on the android platform, which demonstrated well in contrast $t$ o other traditional POQAS systems in a more precise answering providing. Our POQAS-S can be referred to develop more other similar POQAS-S systems besides out built android APP in this p aper.
\end{abstract} robots.

Keywords -Semantics, Knowledge base, Android, and Chat

\section{INTRODUCTION}

Android is a free and open source operating system which is based on Linux, mainly used in mobile devices, such as smart-phones and tablet computers, characterized by Google and Open Handset Alliance. The advantages of Android are: open, rich-hardware, and easy to develop, also the mobile application of Google is a divine place for developers in the heart [1].

Information interaction would never be stopped since the beginning of humanity. Accompanied by the development of social science and technology, it has constantly changed people's form, and always occupies an important position in people's life. As a kind of extension about global information, Mobile terminal network service has greatly changed our life. In today's mobile tide, the mobile terminal as carrier of various service applications is brought to shaking changes our lives [2].

Although there are a large number of information service applications and successful cases on Android platform, due to various reasons, many problems are still existing: the function of Baidu applications is powerful but too popularization and

DOI reference number: 10.18293/SEKE2017-190 not meticulous enough; Twitter has a broad field of information but did not realize information packaging and there are lots of redundant information; the information service of WeChat of the public account is not convenient to find, etc. So, a new, distinctive, concise and powerful for specific groups of APP is in urgent needs.

The purpose of this task is to make up the current information service applications' problems and to absorb their advantages, and developing our application's own characteristics: this application is based on a good interface and with the function that increasing the information storage; In order to satisfy the programmers' demand, adding the push function at the foundation of the reading program articles, using SMS verification functions to make users $\log$ in, which makes the APP easy to use. The "intelligent response to question messages" is the core characteristic.

This design provides an APP for programmers which are compatible with current mainstream features, simple and powerful, focused on providing users with replying related information with high efficiency, reducing the redundancy of searching information, and improving efficiency greatly [3].

With years of development, Android has become a platform, and an ecological system. Now, Android has been widely used in mobile field. In the past, Android versions updated too fast and the compatibility issues make the Android Market share not big, but with its stability improvement and mobile intelligent industry's vigorous development, more and more developers began to enter into the Android world, both in hardware and the software system. All the signs show that, Android is worthy of the name "king of mobile".

Because of the portable mobile devices, application of information service has been rapidly developed. An information service application can help all kinds of people know more about the world and promote people to get the information what they want accurately [4].

In this "data is wealth" era, the success of information servicing applications can bring programmers great opportunities and attract domestic and foreign Pinnacle Technology Corp, free developers, the elite from all walks of 
life. But in the face of huge wealth, competition is more intense compared with other areas.

The main content of this paper is the basic research of a user to an information service application and realize the characteristic functions of the application [5]:

- User management: achieve user registration and log in, retrieve the password,

- messages log in: send the verification code to the phone number, in the time limit of $60 \mathrm{~s}$ and in accordance with the corresponding verification code to $\log$ in the system, the web server can be listening to the corresponding $\log$ in information, the successful logging in is recorded, then when you log in next time, you do not need to enter the verification code.

- Information query: it is the core function, according to the transmitted message, it can find the corresponding answers, which is related to NLP knowledge base. for example, the question encountered in various programs can find answers by this function, also the server can set the information ambiguity [6].

- Article recommended: the server update an article which is related to IT every day, and pushed to the APP's interface, then, the users can view.

- Information storage: the corresponding text, pictures, or video can be stored, viewed and deleted, stored in the SQLite database, and it can use the user's gesture password information to protect.

- Information browsing: the server in the APP in accordance with the classification of the IT technology provides a variety of knowledge encyclopedia information database, users can view the corresponding information; the information database server can be updated in real time.

- Push function: this APP can push the users' mobile phone information in IT aspects.

\section{RELATED WORK}

\section{A. Android technology}

In the domestic mobile phone market, android operating system occupies the most market share. Android based on Java programming language, makes the interfaces and functions take with an endless stream of changes [7].

\section{1) The advantages of Android development:}

a) great openness: Android is opened with source, the system source code can be used by the public, and its modification, distribution is not restricted by the permit.

b) high degree of freedom: the system has a variety of simple and practical widgets, developers can follow their habit to develop application, showing their own ideas and thoughts.

c) advantages of development language: Android use the Java language, because of it, Android development is simple, efficient, rich in resources, and can use lots of frameworks, also with the advantages of connecting with the
WEB server seamless,suitable for current application software industry with increasing competition.

\section{B. Turing engine}

TuringOS is an intelligent operating system which can simulate human feelings and thinking mode. It has the interaction ability which is mostly close to the human, including emotional calculation, thinking and self-learning three engines.

Turing robots have skills to learn so as to suit for all kinds of knowledge fields. Turing engine is based on the NLP knowledge base, leading to artificial intelligence technology and user private knowledge base's effective union. In order to meet the life, and business needs, robot owners can import knowledge bases, and injecting "exclusive content" for their own robot [8].

This APP is based on the engine, with the realization of the function of the core of the question and answer, the difficulty in the programming is the engine's access and design of the APP simulating the box that can accept the online message contents.

\section{REQUIREMENT ANAYSIS}

\section{A. System performance requirements}

Client response: ensure the client's priority with response of user's operations, so as to provide a better user experience; user's $\log$ in, interface conversion and other response time control within $2 \mathrm{~S}$, message receiving and sending response controlling within 1s merely [9].

The server response: with the queue model of request to achieve the effect of response prioritized and treatment delayed, cluster services and provide more stable service; the operation to the database should is control in less than $5 \mathrm{~S}$, manner to the client returns failure information if the operation failed in timely, and rollback data; solve server problem by itself as far as possible, send returning message to the client.

\section{B. System reliability and availability requirements}

Data transmission reliability: To ensure the transmission speed, the conventional data use J-son string transmission; data response reliability: control the way to the database by use of database connection pool technology, reading and writing the database to ensure the efficiency of the database response; client interface usability [10]: clean and tidy interface design, does not appear to provocative color and picture, no embedded advertising, improved functional tips and easy to use;

Menu design usability: reduce the multi-layer nested menu, menu identification is easy to be understood, prefect menu, eliminate the difference of function and instruction; interaction design usability: for a variety of operating, providing a more obvious response, such as text, the box, the progress bar, etc. To avoid stiff interactive or non-interactive, as the first priority, user's response provides a good sense; interface update requirements: the design of the interface configuration by use of form of configuration, easy to modify and follow up; function update requirements: the function of modular design, to ensure that the system's high scalability, easy to follow up function; server needs to expand the server: when the client sends a request to the server, by forward the request to intercept 
and forward the request to adapt to the subsequent expansion; database design requirements: the table of the database and the field obey unified standard design, in order to follow the increasing amount of data, it may be used to separate the implementation of reading and writing [11].

\section{Feasibility analysis}

Analysis of the feasibility of the task is the necessity and feasibility of the application of project development. Necessity comes from the urgency of the task, but feasibility depends on the realization of the application system of the resources and conditions. The project need set up on the basis of preliminary investigation.

\section{Technical feasibility}

Android is one of the most popular mobile terminal platform, it has good usability and ease of development, resource rich and so on. At the same time, many incomparable advantages for developers to provide strong function and the abstraction, so that developers can avoid repeated low-level work and focus on business and achieve.

Java, as one of the most widely used business language, has the characteristics of high efficiency, stability and rich open source framework. It plays an irreplaceable role in server programming.

This project uses the Android client, and servers which are based on the Turing robot engine. Being use of interface framework it can be an architecture level of good design of the system [12]:

- Client as the view display layer, based on the use of Eclipse Java prepared. On the one hand, Android platform for the development is simple and efficient. on the other hand, rich open source information of Android platform can provide us with a powerful boost;

- As control layer, Server-side logic part based on eclipse using Java, through the use of Turing robot engine server can make our development focus on business. on the other hand, Java, with the stable and efficient merit and rich and open framework, with the popularity of spring and Struts2 framework which greatly facilitated the development to us;

- Above all, the client uses the Android platform application development to realize high efficiency, on the other hand, with the help of Turing engine and open source framework, the server can gain a robust architecture and efficient development, in terms of technology, it is feasible [13].

\section{E. Economic feasibility}

The cost of this project included two parts: the cost of the hardware device such as a server and the mobile terminal hardware such as a test machine.

At present, a personal PC, personal mobile terminal intelligent equipment is enough to meet the requirements. As college students, these two devices can be very convenient to get in, even if the repurchase, the burden of cost is not great. And application software system, due to the characteristics of
Java and Android, a large number of excellent open source frameworks and the servers can allow us to use it free without charge, so the cost are allowed to ignore in this hand. After the completion of the project, we can through upload app to store and get download traffic revenue consequently [14].

\section{F. Risk factor control feasibility}

As a project, this project can access to information from the knowledge base and Internet when meeting some mistakes, which to ensure that the project is without errors.

\section{POQAS-S SYSTEM BASED ON ANDROID}

\section{A. Overall design}

Overall design is mainly to transform the logic model of demand analysis into the physical model of the overall design, and set out to achieve the demand of the system. Overall design is not only to design the system of the overall function structure, but also the relationship between these modules and signals between them. Because modules are independent of each other, so we can separate the processing such as code design, write, test and modify, thus reducing the possibility of error spreading in the module, improving the reliability and maintainability of the system. And then for the design of database, which is an important part of the system, a good database can make the system run more smoothly. At the beginning of the development of this system, we should design each data set in the database, and the standard relationship between them.

\section{B. System function design}

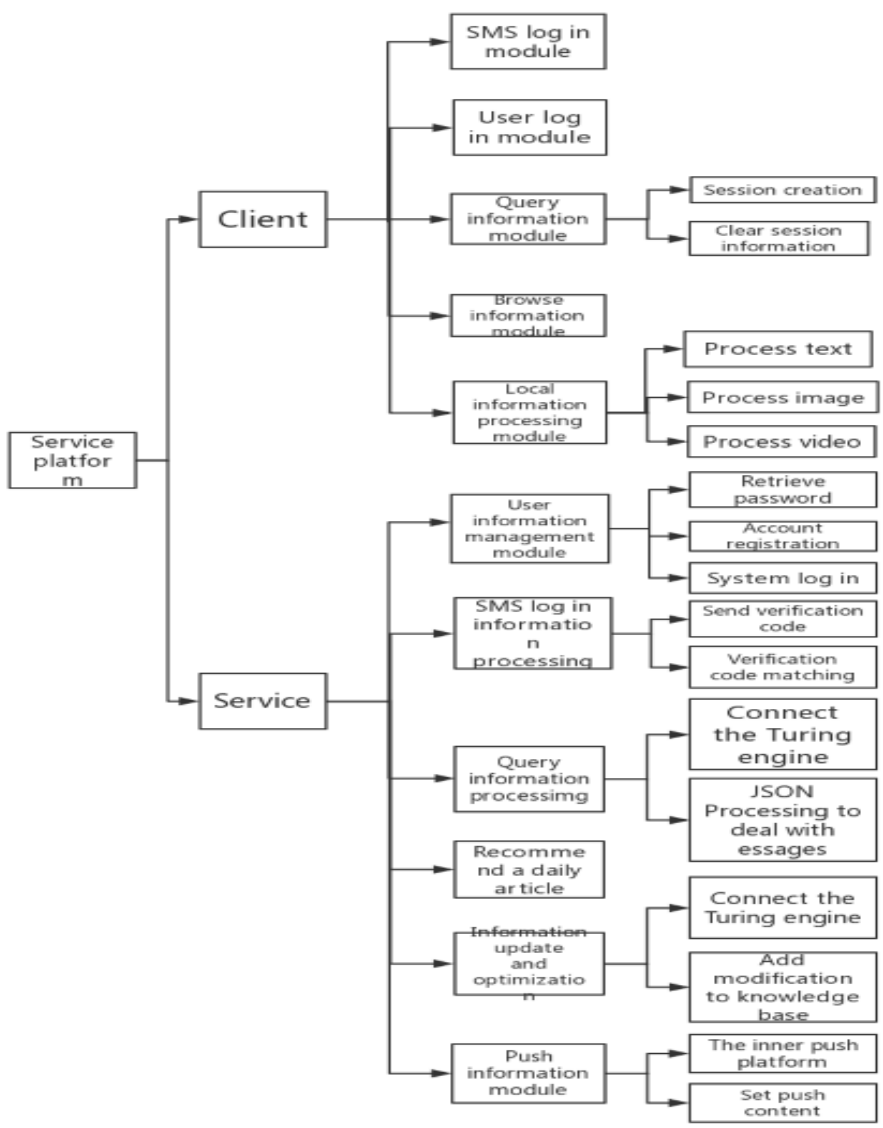

Figure 1. The system function module chart 


\section{Android client}

\section{1) Query information module}

The module is mainly responsible for sending request information. Getting request information from the client activity, changing it into corresponding Json string format, and then these strings assembly to the corresponding URL parameter way. The HTTP can get access to this URL and send the information to the server.

\section{2) Browse information module design}

The module is mainly responsible for the server to receive and parse information.

On the server side, the results transported by $j$-son strings (transformed by $g$-son object) are complied with the HTTP agreement. The client gets the corresponding $j$-son string from input-stream complied with the HTTP agreement, definitely the $j$-son string also transformed to the corresponding java class object. The parsing process needs external jar package ( $g$-son package). The corresponding Java objects are used for displaying users' information on the client side.

\section{3) Local information storing module}

The module is mainly responsible for managing the information stored on the client side, such as adding, deleting, and modifying, inquiry and etc. information including text, pictures, videos can be stored for users. Users can call the camera and videos hardware interface on the client side to save the information they wanted, and to remark the information which is stored in the Sqlite database, and by generating the related thumbnail in the style of list view. So, users can check and delete information stored at any time.

In order to ensure the security of stored information, Gesture passwords lock is used in this module. Users can set the gestures passwords before they used. At the next time, when users use the module, they must be verified by gestures passwords. Of course, when you are verified successfully, it can also be closed. Gesture passwords have set the boot menu, making users setting conveniently, and through this interface by setting up the boot sequence of gesture passwords graphics, user-friendly and beautifully.

\section{4) Information processing module}

a) Users logging in module

The client is responsible for the judgment of inputting information which is legal or not, the system is responsible for the information verifies, especially for determining whether the inputting information matched completely the information in the database. If the information is correct, it will be transformed to a user class object, which is through the socket transmissions, sending logging request to the server. Server will parse the user object of user information, and then check the information of the user existing or not, if the user information exists, then the server returning the message that is logging in successful to the client. The client jumps to the next Activity. If the user information d does not exist, then the server returning to the client: the user name or password error. The client prompts users to input the user information again.

\section{b) SMS landing module}

Users can use the phone number, and then enter the verification code, its limit time is $60 \mathrm{~s}$, while, if the information is matched, this action is successful; otherwise the system pop the message box to ask users whether or not to send verification code again. If there are records of a successful logging in, the mobile phone number will be preserved, and users can skip the stage of verification code next time.

The function named setRegisterCallback is responsible for handling the response of the related event. Firstly defining a RegisterPage class which is imported by an external SDK package, but method named afterEvent must be rewritten: if the country and the phone numbers are inputted, they will be uploaded to the SMS by using the method of hash map server, and submitUserInfo upload function also needs to be rewritten: The function named submitUserInfo needs to be added two additional parameters named registering nickname and random UID (using mathematical random function).If the verification is successful, then jumping to the main interface of the app, and saving the information successfully. If the validation fails, jumping back to the logging interface again [15].

\section{c) Update information and optimize the module}

After logging in the system, the designer can update the knowledge base which is of knowledge module, and the existing knowledge base module including data structure, algorithm, programming language, computer basis, application software system development, mathematical and logical ability module can be altered. The changing of corresponding modules is side in Turing engine, which is the server side. For instance, modifying the server NLP knowledge base can realize the information update.

\section{d) Push information module}

The module is mainly responsible for information recommendations. Developers will put the important programming information as a hot topic to users who use this app. The specific push messages are defined in the platform of JPUSH garage, through an external SDK to use the platform to implement the corresponding information push operation, this process plays an important role for users to increase the programming knowledge which is the core for the aim of this system.

\section{e) Recommended daily article module}

The server will update information of corresponding NLP knowledge base every day to achieve the requirement of a good programming article which is recommended daily, the corresponding good articles were collected, and added in the NLP knowledge base. Users will transmit the request information encapsulated good daily article to the server, and then, the server obtains the information, and queries the corresponding of NLP knowledge base. Note: due to the specified format returned and the length of paper generally are too long, so we should be created the additional buffer pool space to realize the information buffer [16]. There are two ways to realize the article pushed, one is using the existing JPUSH platform to display on the client, the other is using Turing engine to push.

\section{f) Socket transmission}


Using the object of class of the Socket to write data in the input process. Firstly, server-side declares a Server-Socket object and specifies the port number, and then call the accept method of Server-Socket to receive the client's data. When there is no received data, the method accept is in blocked state. Once receiving the data, the method read deal with the received data through the input-stream. The client creates a Socket object, which specifies the server IP address and port number to read the data from the input-stream, by this, the client obtained the data from server. Finally, Client sends the data which should be sent to the output-stream. Namely it is time to precede socket data transmission of TCP protocol. Server-side logic process: when the information of users who $\log$ in is empty, the user object which the server obtained will return the prompt message of empty logging information. Otherwise, according to the logging information submitted by user object, Server-side invoke $\log$ method of server-side method User-Dao to log in.

\section{g) Registering}

In this module, when the user firstly enters the logging page, he must fill in the blanks which are "user name", "phone number", "password" and "confirm password", "your spouse or father or mother or children or company boss or your primary school teacher's name". The client access to these information, firstly checkout the password in the third blank is same with the second blank. if it is not consistent, then the client reminding the user to fill in it again. The server would get the information until the client conforms the information to meet the requirement of the standard.

\section{h) J-son parsing module}

The module is mainly responsible for the $J$-son object which is to deal with a request transformed from Client parsed into Java objects, Server obtains $J$-son object from the client request information, according to the current request interface set to find the corresponding tools in this module, which parsing the $J$-son object to a given Java object, so as to give back the other requests interface to use.

\section{CONCLUSION}

This paper introduces an APP created by us. The system was basically completed, but due to the writer is still insufficient in terms of technology and design ability, some function implementation could be optimized. Firstly, the pushing information module could be changed by using the latest frame. Secondly, the knowledge base could use k nearest neighbor algorithm in machine learning to improve the match rate in searching. Most importantly, more measures should be used to check the safety of our system because we are more interested in speed than safety and reliability.

According to our experiments on questions in programming area to be asked to our system, it achieves $85 \%$ right solved rate (comparable to other POQAS systems) and $1.5 \mathrm{X}$ faster than these systems, which makes it the great solution viable for use cases of POQAS systems. But we still need to update knowledge base constantly to improve the right solved rate,

\section{ACKNOWLEDGEMENT}

This work has partially been sponsored by the National Science Foundation of China (No. 61572355) and Tianjin Research Program of Application Foundation and Advanced Technology under grant No. 15JCYBJC15700, Xinjiang Corps Technology Market Association project under grant No. 2016GKF-0693.

\section{REFERENCES}

[1] Zong Woo Geem, Joong Hoon Kim, G. V. Loganathan, "A new heuristic optimization algorithm: Harmony search,” in SIMULATION, vol. 76(2), pp. 60-68, February 2001.

[2] Moles CG1, Mendes P, Banga JR, "Parameter estimation in biochemical pathways: a comparison of global optimization methods," in Genome Research, vol. 13(11), pp. 2467-74, 2003.

[3] Alexander Budanitsky, Graeme Hirst, "Evaluating WordNet-based Measures of Lexical Semantic Relatedness," in Computational Linguistics, vol. 32(1), pp. 13-47, 2006.

[4] Alan Aronson, "Effective Mapping of Biomedical Text to the UMLS Metathesaurus: The MetaMap Program," in Proceedings of the AMIA Symposium, pp. 17-21, 2001.

[5] YM Wang, TMS Elhag, "Fuzzy TOPSIS method based on alpha level sets with an application to bridge risk assessment," in Expert Systems with Applications, vol. 31(2), pp. 309-319, 2006.

[6] Huan Liu, Lei Yu, "Toward integrating feature selection algorithms for classification and clustering," in IEEE Transactions on Knowledge and Data Engineering, vol. 17(4), pp. 491-502, April 2005.

[7] A Goldsmith, SA Jafar, I Maric, S Srinivasa, "Breaking Spectrum Gridlock with Cognitive Radios: An Information Theoretic Perspective," in Proceedings of the IEEE, vol. 97, no. 5, pp. 894-914, May 2009.

[8] Clément Sanchez, G. J. de A. A. Soler-Illia, François Ribot, T. Lalot, Cédric R Mayer, V. Cabuil, "Designed Hybrid Organic-Inorganic Nanocomposites from Functional Nanobuilding Blocks," in Chemistry of Materials, vol. 13(10), pp. 3061-3083, 2001.

[9] Igor Luzinov, Sergiy Minko, Vladimir V Tsukruk, "Adaptive and responsive surfaces through controlled reorganization of interfacial polymer layers," in Progress in Polymer Science, vol. 29(7), pp. 635698, 2004.

[10] Uttam C. Paul, Despina Fragouli, Ilker S. Bayer, Athanassia Athanassiou, "Functionalized Cellulose Networks for Efficient Oil Removal from Oil-Water Emulsions," in Polymers, vol. 8(2), pp. 52, February 2016.

[11] Ling Wang, Quan Li, "Stimuli-Directing Self-Organized 3D LiquidCrystalline Nanostructures: From Materials Design to Photonic Applications," in Advanced Functional Materials, vol. 26(1), pp. 10-28, November 2015.

[12] Zan Guangtao, Wu Qingsheng, "Biomimetic and Bioinspired Synthesis of Nanomaterials/Nanostructures," in Advanced Materials, vol. 28(11), pp. 2099-2147, 2016.

[13] Kumar Dipesh, Chatterjee Kalyan, "A review of conventional and advanced MPPT algorithms for wind energy systems," in Renewable and Sustainable Energy Reviews, vol. 55, pp. 957-970, March 2016.

[14] Holtgraves Thomas, Han Tai-Lin, "A procedure for studying online conversational processing using a chat bot," in Behavior Research Methods, vol. 39(1), pp. 156-163, February 2007.

[15] Imran Ali Shariq, Kowalski Stewart James, "HIP - A Technology-Rich and Interactive Multimedia Pedagogical Platform," in Lecture Notes in Computer Science, vol. 8523, pp. 151-160, 2014.

[16] Kang Ah Reum, Kim Huy Kang, Woo, Jiyoung, "Chatting pattern based game BOT detection: Do they talk like us?" in KSII Transactions on Internet and Information Systems, vol. 6(11), pp. 2866-2879, 2012. 